\title{
ASSESSMENT OF POINT CLOUD ANALYSIS IN IMPROVING OBJECT-BASED AGRICULTURAL LAND COVER CLASSIFICATION USING DISCRETE LIDAR DATA IN CABADBARAN, AGUSAN DEL NORTE, PHILIPPINES
}

\author{
T. A. M. Rollan ${ }^{1}$, A. C. Blanco ${ }^{1,2}$ \\ ${ }^{1}$ Phil-LiDAR 2, UP Training Center for Applied Geodesy and Photogrammetry \\ ${ }^{2}$ Department of Geodetic Engineering, College of Engineering \\ University of the Philippines - Diliman, Quezon City, Philippines - therese.rollan@gmail.com, ariel.blanco@coe.upd.edu.ph
}

KEY WORDS: agriculture, land cover, LiDAR, point cloud analysis, object-based image analysis, OBIA, eCognition

\begin{abstract}
:
Cabadbaran City is the capital of Agusan del Norte which is located at the north eastern portion of Mindanao, Philippines. Almost $30 \%$ of its land area is devoted to agriculture (mainly rice, corn, coconut, banana, vegetables and abaca). Currently, the city government and agriculture office are implementing programs focusing on improving coconut and vegetable productivity, controlling banana disease and infestation, and enhancing abaca production industry. In support of decision making, the current situation must first be assessed by answering the basic questions what and where through detailed and accurate resource mapping. In this study, only discrete LiDAR datasets were utilized. Corresponding orthophotos were used only for training and validation. Land cover classification was done using two workflows using Support Vector Machines (SVM) as the classifier. In the first workflow, land cover classes were classified using rasterized point cloud metrics such as minimum, maximum, standard deviation, skewness, kurtosis, quartile average, mode and median. In the second workflow, point cloud analysis was used to derive additional features for classification which was integrated and executed in the same object-based software through Cognition Network Language $(\mathrm{CNL})$. The derivations of the additional features were conducted after running an initial segmentation which means that the distribution of points was analysed within the delineated objects. Classes that benefited to point cloud-based metrics are mostly non-ground agricultural classes namely coconut, mango and palm trees. These classes have obtained increase in accuracies by an average of $11.2 \%$ using validation sample set 1 and an average of $18.2 \%$ using validation sample set 2 . Ground classes, particularly barren land and rice, appeared to be incompatible to these point cloud metrics as shown by the decrease in accuracies for Methods 2 and 3 by about $18.1 \%$ using validation sample set 1 and about $16.4 \%$ using validation sample set 2 . Exploring other useful point cloud-based metrics and testing on sites with other land cover classes are highly recommended.
\end{abstract}

\section{INTRODUCTION}

Agriculture is vital in every country's food security. It supports the people's daily needs not only in terms of food but also in providing raw materials essential for the production of medicines, clothing, and many more. In the Philippines, the agricultural sector feeds more than a hundred million citizens (Philippine Statistics Authority, 2016) which most of the time is said to be unable to provide sufficient supply of goods particularly rice. Some of the reasons are the lack of allotted area for plantation, scarcity of water during the dry season that leads to lower yields and higher production cost caused by a number of typhoons that hit the country (Dy, 2015). Because of these, there is a need to import goods to fill up these gaps. A way to deal this is to monitor and plan the yields and the allotment of area for the sector such that the volume of yields will fit the intensity of demand. There should also be a way to monitor and regulate the conversion of land use from agricultural to residential or commercial. In order to do these, the sector must know the current status of the crop areas starting from the basic questions what and where. What crops are being cultivated and where are they situated? These can be answered by resource mapping. Resource maps are maps showing specific type/s of target land cover/s. A variety of methods can be conducted to produce a resource map. In this study, two methodologies will be presented using LiDAR technology and object-based image analysis (OBIA).

\section{RELATED LITERATURE}

\subsection{Point Cloud Analysis and Applications}

LiDAR (Light Detection and Ranging) is a form of a remote sensing system of acquiring data by radiating a pulsed laser to obtain measurements to the earth (National Oceanic and Atmospheric Administration, 2015). At a given target, distance from the platform can be calculated knowing the speed of the laser pulse and the time it takes for the pulse to return to the sensor (LiDAR-UK). A GPS (Global Positioning System) and Inertial Measurement Unit provide position and orientation information to the system respectively (Bao, et al., 2008). The output point cloud data consist of 3D coordinates $\mathrm{x}, \mathrm{y}$ and $\mathrm{z}$ and Intensity i. Based on these four attributes, various rasters can be derived such as Intensity, Number of Returns, and Digital Elevation Model (DEM) images (Carranza, et al., 2014). Moreover, from these images, we can further derive height textures and metrics. In the study conducted by Qiao and Zhang (2009), height and intensity textures were used to classify a LiDAR dataset into five classes: cropland, bare land, water body, man-made constructions, and sparse tree land. These textures include mean, entropy, variance, second moment and homogeneity that served as criteria for the Artificial Neural Network (ANN) classifier. Aside from land cover mapping, LiDAR point cloud can also be used for environmental management. One example is how Peterson et al. (2005) predicted canopy bulk density (CBD) and canopy base height $(\mathrm{CBH})$ for a portion of the Sierra National Forest, Nevada. Moreover, they also used LiDAR data for canopy fuel mapping 
which served as input for a fire behaviour model (FARSITE). Kandrot (2013) tackled a new approach and its advantages on coastal monitoring particularly the dynamics of sand dunes morphology. More uses of point cloud analysis include urban (Harrap \& Lato, 2006), disaster mitigation (Disaster Risk and Exposure Assessment for Mitigation (DREAM) Program, 2016), and medical applications (Schultz \& Kindlmann, 2013).

\subsection{Support Vector Machines (SVM)}

Support Vector Machines (SVM) is a type of supervised classification in which a hypothesis space of linear functions in a high dimensional feature space (Cristianini \& Shawe-Taylor, 2000). According to Cristianini and Shawe-Taylor (2000), SVM algorithm finds a way on obtaining efficient separating hyperplanes such that the generalization bounds are optimized.



Figure 1. Simple Illustration of How SVM works as adapted from Burges (1998)

Despite its promising and very strong theoretical background, it remained unnoticed in its early publications (Kecman, 2005). The user may tweak on its parameters upon using it to certain application. These include $\mathrm{C}$ or the penalty factor (Alpaydin, 2014), Epsilon or the value that indicates the percentage or ratio of the support vectors to the number of samples (Mattera \& Haykin, 1999), Gamma which defines the extent of influence of a training sample (scikit-learn.org) and Kernel functions which are used to project non-linearly separable samples into linear separable feature space (Hofmann, 2006). There are four types of kernel functions namely Linear, Polynomial, Radial Basis Function (RBF) and Sigmoid (McCue, 2009). Kecman mentioned in his book section that it is only when practical applications yielded excellent results that SVM became known and accepted. These applications include digit recognition, computer vision and text categorization. For land cover classification, Zhang, Lin and Ning (2013) utilized SVM for airborne LiDAR point clouds in urban areas. Roli and Fumera (2001) introduced and applied SVM with varying parameters to multisensory images and compared its performance to Multilayer Perceptrons Neural Network and k-NN classifiers. Their output classes include sugar beets, stubble, bare soil, potatoes and carrots. A data fusion was done by Waske and Benediktsson (2007) using multitemporal synthetic aperture data and optical imagery. Their proposed SVM-based fusion methodology had significantly increased the accuracy results of a single run of SVM consisting classes namely arable crops, cereals, canola, root crops, grassland, orchard, forest, and urban areas.

\section{MATERIALS AND METHODOLOGY}

\subsection{Study Area}

Agusan del Norte is situated at the south-eastern part of the Philippines. The province's major crops include rice, corn, coconut, abaca, banana and mango (Provincial Agricultural Office of Agusan del Norte). A portion of the municipality of Cabadbaran approximately located at $9^{\circ} 06^{\prime} \mathrm{N}, 125^{\circ} 34^{\prime} \mathrm{E}$ was selected as the study site which covers an area of about 6 sq. $\mathrm{km}$. It is comprised of land covers built up areas, water body, grassland, bare land, rice, corn, non-agricultural trees, coconut, mango, banana and oil palm trees. A banana plantation is located at the leftmost portion while mango plantations are distributed within the study area. Coconut and oil palm trees are often located in the same area. Corn parcels contain sparse plants but can still be identified.
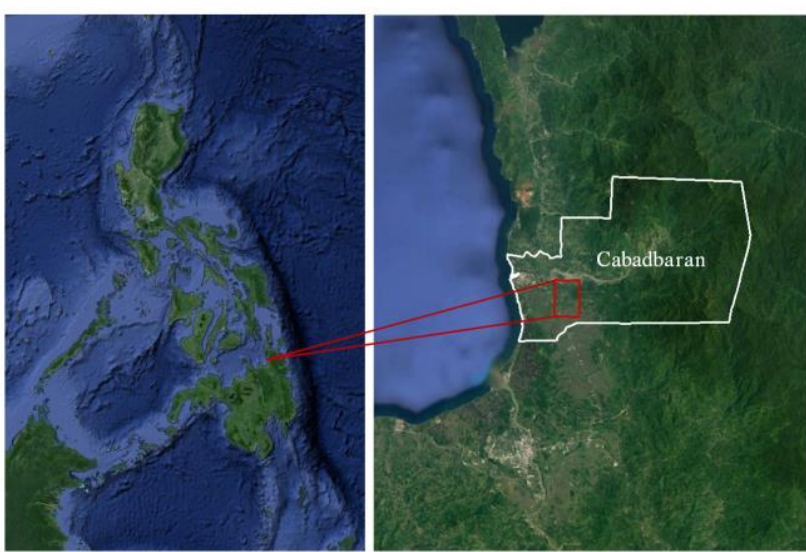

Figure 2. Study Area (Google, 2012)

\subsection{Datasets}

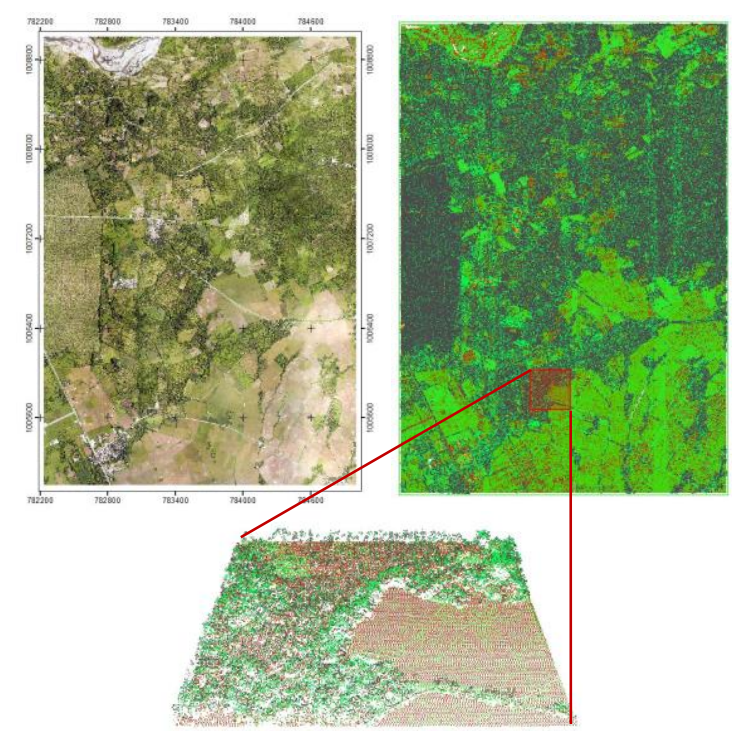

Figure 3. Datasets: Orthophoto (left), Point Cloud (right) and Point Cloud Perspective View (bottom)

The data used include an orthophoto with $0.5 \times 0.5 \mathrm{~m}$ resolution and a pre-classified LiDAR point cloud (.las) with 2 points per sq. km. resolution. The orthophoto contains three bands: Red, Green and Blue. The point cloud was pre-classified into general classes: ground, vegetation (low, mid and high), and buildings. 


\subsection{Methodologies}

Three classification methodologies were applied and corresponding results were compared. The first method utilized raster-based derivatives (see Figure 4), the second used point cloud-based derivative layers, and the third is a combination of the two sets of derivatives. Orthophotos are available but were only utilized for the selection of training and validation points as this research is on the classification of agricultural cover using LiDAR data only.
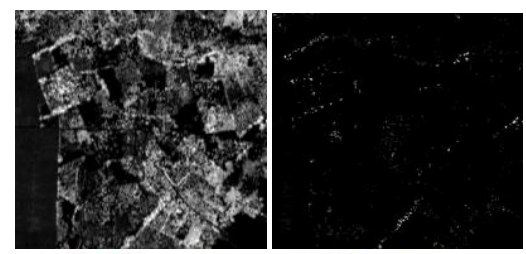

Height Average Value

High : 31.6

Low: 0



Value

High : 20.96

ow : 0

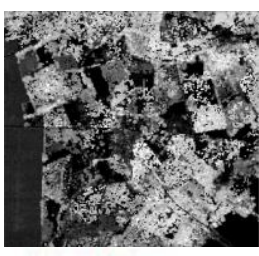

Height Maximum

Value

High: 37.8

ow: 0
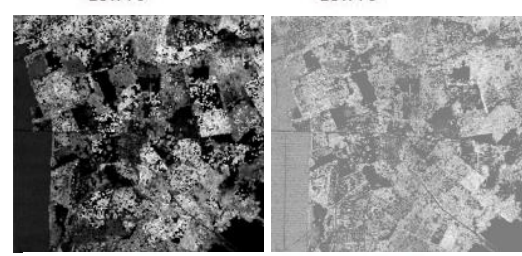

Height Std. Dev.

Number of Returns

Value

High: 15.74

Low : 0

High : 4

Low: 1
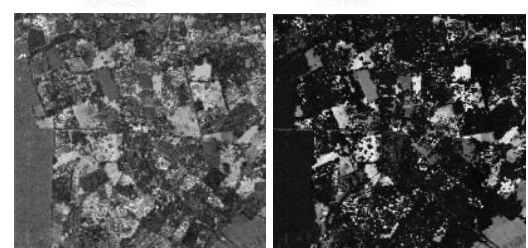

Intensity Average

Intensity Minimum

Value

Value
High: 179

High : 17

Low: 3

Low: 0

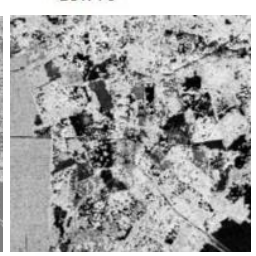

Slope 2x Exag

Value

High: 89.0411

Low:0

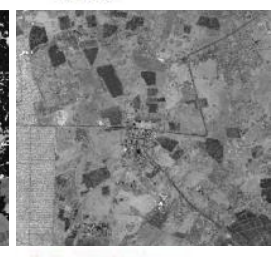

Intensity Maximum

Value

High : 255

Low : 3


Intensity Std. Dev.

Intensity Median

High: 86

,

Low: 0
Intensity Highest 1st Returr Value

How

Low: 1

Figure 4. Examples of LiDAR Derivatives used

Figure 5 illustrates the classification workflow for method 1 in which height and intensity metrics were calculated per specified pixel size. A relatively large pixel size of 5 x 5 meters was used to be able to feed enough number of points for the computation of the metrics (Jerez, et al., 2015) using Lastools. LiDAR derivatives include normalized Digital Surface Model (nDSM) or the height image, intensity, slope, number of returns, height metrics (average, minimum, maximum, standard deviation, mode and median or the $50^{\text {th }}$ percentile), and intensity metrics

(average, minimum, maximum, first returns maximum, last returns minimum, standard deviation, mode and median) (see Figure 4). These derivatives were then imported in eCognition where initial segmentation was done using nDSM to separate non-ground from ground features. Non-ground and ground objects were further segmented using nDSM and intensity respectively until meaningful objects were obtained. Meaningful objects indicates no multiple type of feature are in the same object. Moreover, minimal over-segmentation (i.e., single type of feature were broken into smaller pieces) was committed.

Training points were selected using the orthophoto. Separate SVM classifications for the two general classes were conducted in order to avoid confusion between low and high vegetation (Jerez, et al., 2015). The parameters that were used include $1 /$ (number of dimensions or the derivatives used) for the Gamma parameter which is the default value, 200 for the C parameter, and RBF kernel function (Carranza, et al. 2014). Output final classes include water body, grassland, bare land, rice and corn for ground and built up areas, non-agricultural trees, coconut, mango, banana and oil palm trees for nonground.

Validation samples for the accuracy assessment were then selected such that they do not overlap with the training samples and the number of samples follows the ratio $70 \%$ training - 30\% validation (Jerez, et al., 2015). From the same reference, individual accuracies especially for the agricultural classes were examined and necessary improvement was done in order to meet the minimum required accuracy of $85 \%$ set for classification using LiDAR dataset only (no orthophoto).

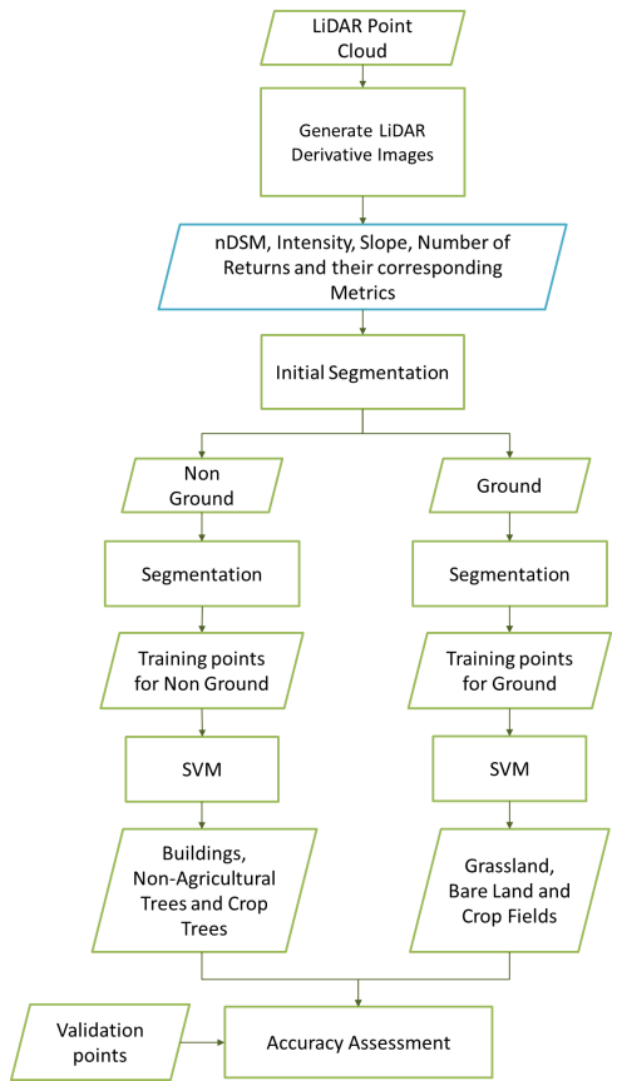

Figure 5. Method 1: Using Raster-Based Metrics for Land Cover Classification 
In the second methodology, height and intensity metrics were computed directly from the point clouds per object. Figure 6 shows the workflow in which deriving the metrics (in blue box) were not done prior to the whole classification procedure. The same set of ground and non-ground objects were used in the first two methodologies to ensure that the differences in the output will only be caused by the difference in the computation of the metrics. In the third methodology, derivatives from Methods 1 and 2 were combined.

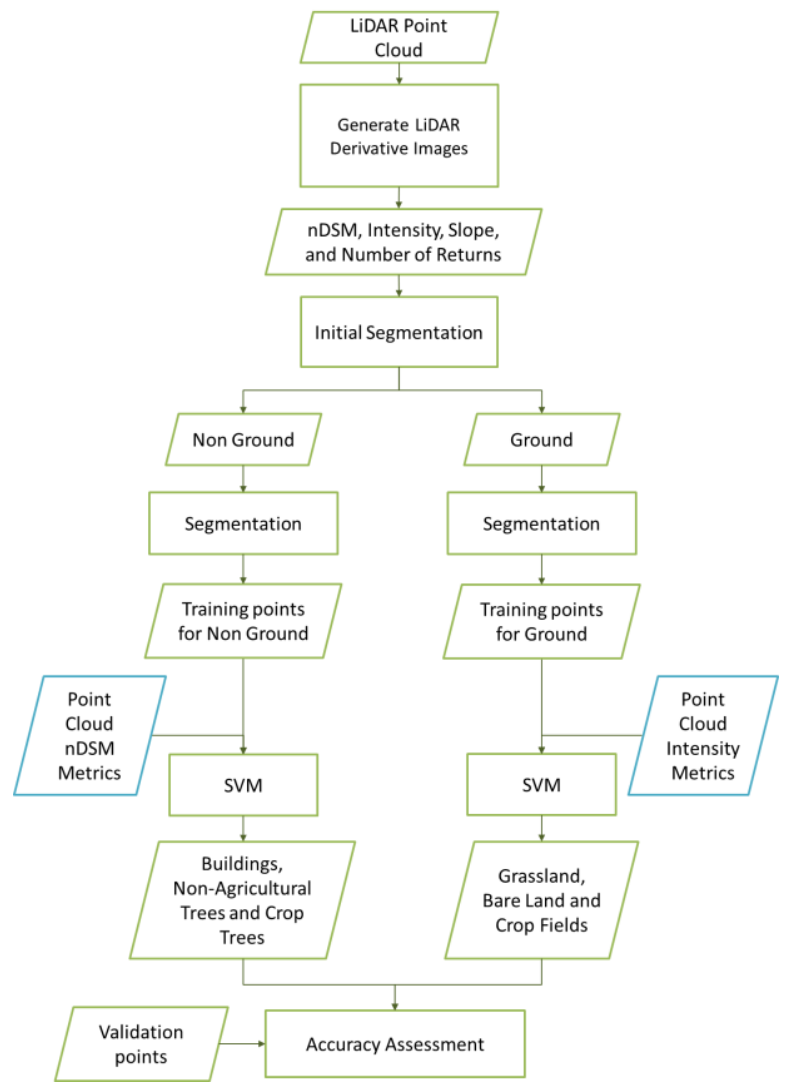

Figure 6. Method 2: Using Point Cloud-Based Metrics for Land Cover Classification

Lastly, the output accuracies from the three methodologies were compared per class. Two sets of validation samples were obtained in order to check the consistency of the results.

\section{RESULTS AND DISCUSSION}

Final classes include barren, corn, grassland, rice, banana, coconut, palm, mango, non-agricultural trees and buildings. The classification results for the three methodologies were visually examined. To quantify the differences among results, accuracy assessment was conducted using two sets of validation samples. Trends of the accuracy values were also observed per class from method 1 to 3 . Figure 7 shows the classification outputs from the 3 methodologies. Some portions of the classified area are presented in Figure 8.

By visual inspection, differences are not too apparent except for rice and barren. Methodologies 2 and 3 have similar classification outputs as compared to that of Methodology 1 . The banana plantation located at the left side was captured by the three methodologies and differs at some parts only. At the lower right of the classified images, the differences are most apparent where barren and rice parcels are mostly located. Method 1 had detected the least area of rice parcels and, consequently, the largest barren areas.

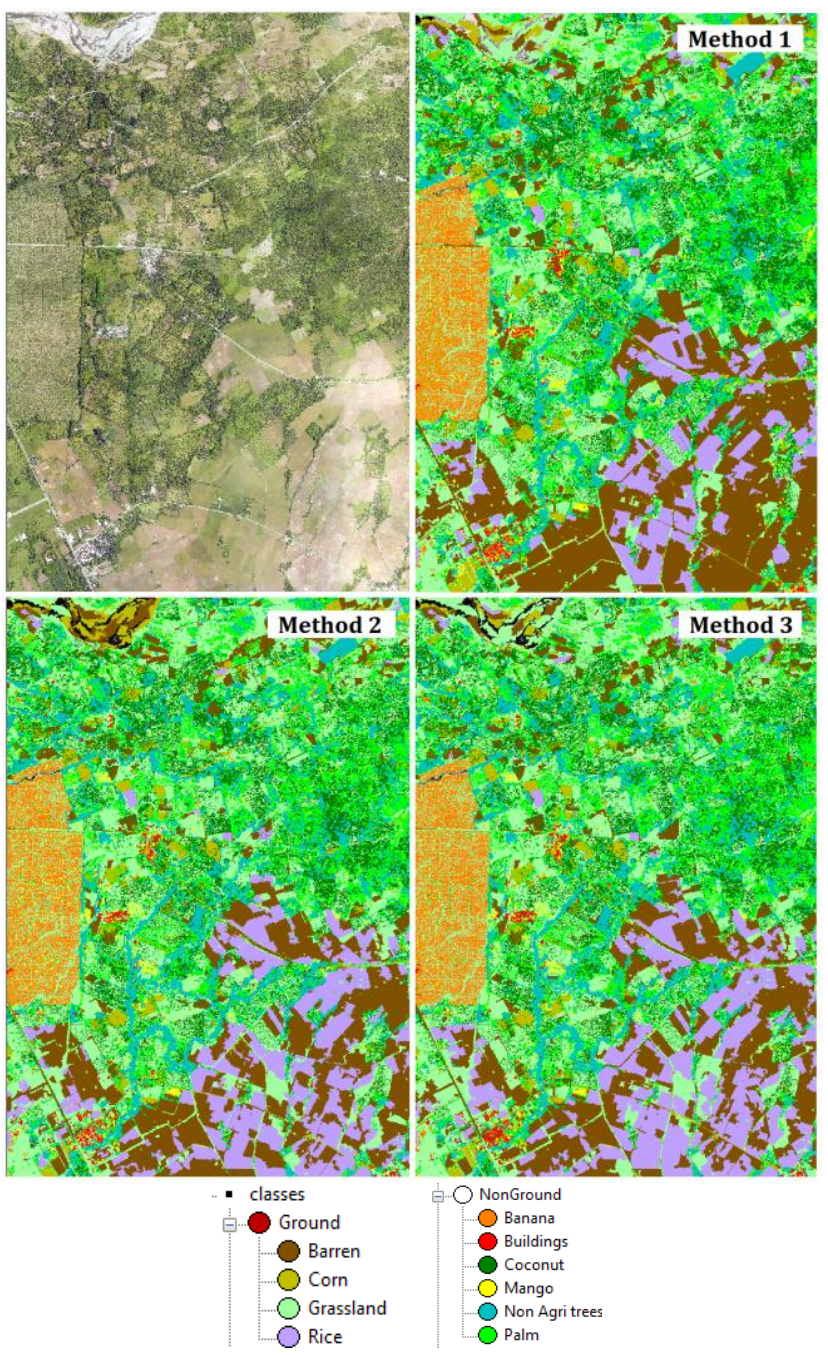

Figure 7. Orthophoto (upper left) and Classification Results for Methods 1, 2 and 3

The top three highest accuracies for validations sample set 1 are from corn (1.000 from all methods), banana (1.000 from all methods), and grassland (1.000 from Methods 1 and 3) while for validation sample set 2 are grassland, corn, coconut, banana, and buildings ( 1.000 from all methods). On the other hand, the top 3 lowest accuracies from validation sample set 1 are from mango (0.588 from Method), barren (0.661 from Method 2), and rice (0.705 from Method). For validation sample set 2, top 3 lowest accuracies are from rice ( 0.451 from Method 2), barren (0.540 from Method 2), and mango (0.651 from Method 1). Method 1 yielded the highest overall accuracy of 0.944 and Method 2 yielded the lowest overall accuracy of 0.771 . 

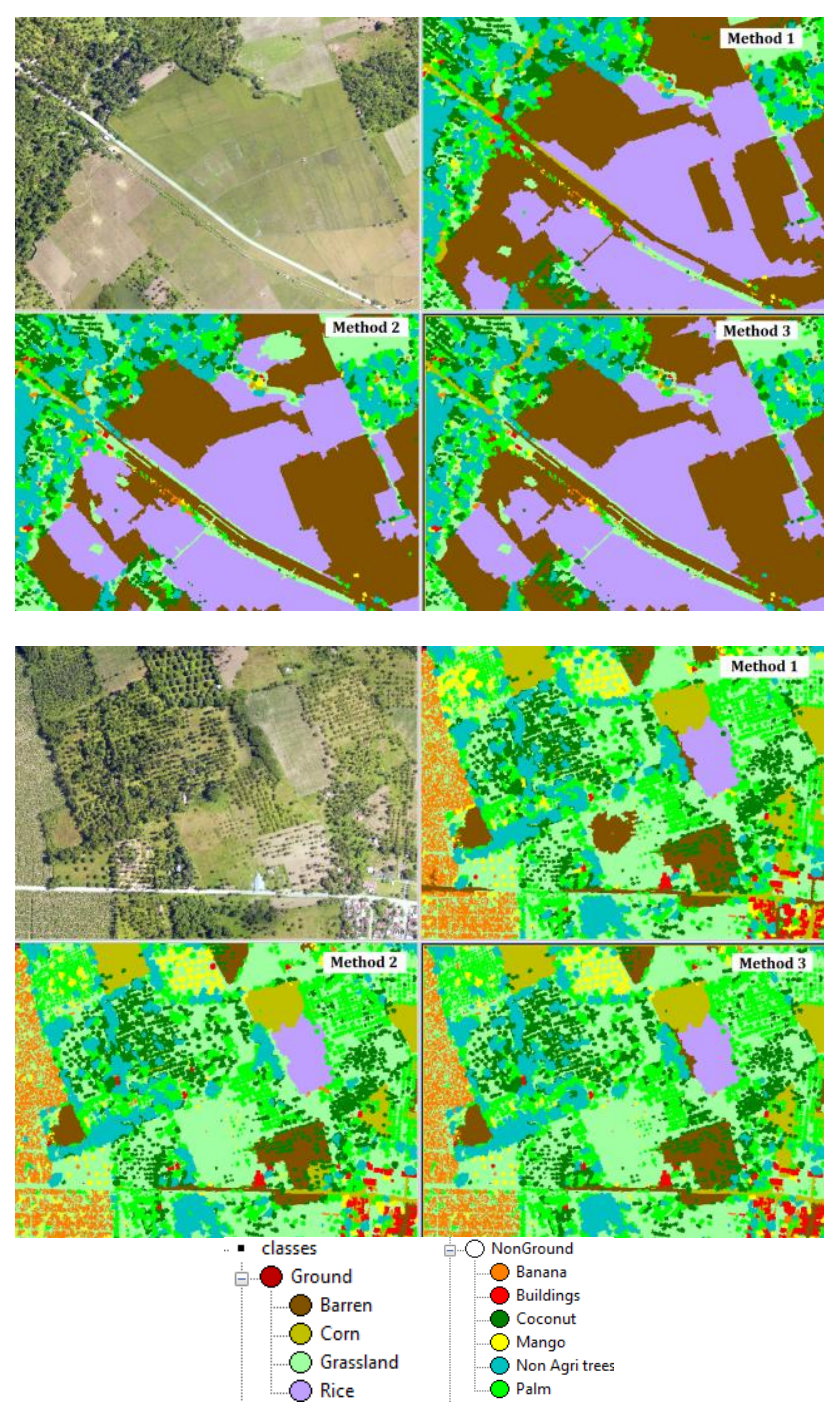

Figure 8. Portion of the Classification Result for Methods 1, 2 and 3

Observing the trend of accuracies for validation samples set 1 from Method 1(raster-based) to 2(point cloud-based) (Table 1 and Figure 9), accuracies of three (Coconut, Mango and Palm trees) out of ten classes have increased values, four (Barren, Grassland, Rice and Non-Agricultural trees) classes and the Overall Accuracy obtained lower values and the remaining (Corn, Banana and Buildings) have retained their accuracy level. On the other hand, observing the accuracies using validation sample set 2 from Method 1 to 2 (Table 2 and Figure 10), two classes (Mango and Palm Trees) obtained increase in accuracy values, three classes (Barren, Rice and NonAgricultural trees) and the Overall Accuracy have obtained decrease in accuracies, and five (Grassland, Corn, Coconut, Banana and Buildings) have retained their accuracy level.

Comparing the results of processing the two sets of derivatives (Method 1: raster- and Method 2: point cloud-based) separately to the combined derivatives (Method 3) using validation set 1 (Table 1 and Figure 9), the classification of the following classes improved with Method 3: Grassland, Mango, Palm and Non-Agricultural trees. However, Barren land, Rice, Corn, Coconut, Banana, Building and the Overall Accuracy did not obtain any increase in accuracy. For validation sample set 2
(Table 2 and Figure 10), accuracy improvement was observed for the Barren land, Rice, Mango, Non-Agricultural trees classes, as well as in the Overall Accuracy. For the remaining classes namely Grassland, Corn, Coconut, Banana, Palm, and Buildings, no increase in accuracies were obtained.

Table 1. Accuracy Results of Validation Sample Set 1

\begin{tabular}{|l|c|c|c|}
\hline \multirow{4}{*}{ Class } & \multicolumn{3}{c|}{ Method } \\
\cline { 2 - 4 } & $\mathbf{1}$ & $\mathbf{2}$ & $\mathbf{3}$ \\
\hline Barren land & 0.872 & 0.661 & 0.620 \\
\hline Grassland & 1.000 & 0.985 & 1.000 \\
\hline Rice & 0.935 & 0.785 & 0.705 \\
\hline Corn & 1.000 & 1.000 & 1.000 \\
\hline Coconut & 0.976 & 0.979 & 0.930 \\
\hline Banana & 1.000 & 1.000 & 1.000 \\
\hline Mango & 0.588 & 0.831 & 0.957 \\
\hline Palm & 0.774 & 0.864 & 0.812 \\
\hline Non-Agricultural Trees & 1.000 & 0.966 & 0.981 \\
\hline Buildings & 0.851 & 0.851 & 0.851 \\
\hline Overall Accuracy & 0.944 & 0.854 & 0.829 \\
\hline
\end{tabular}

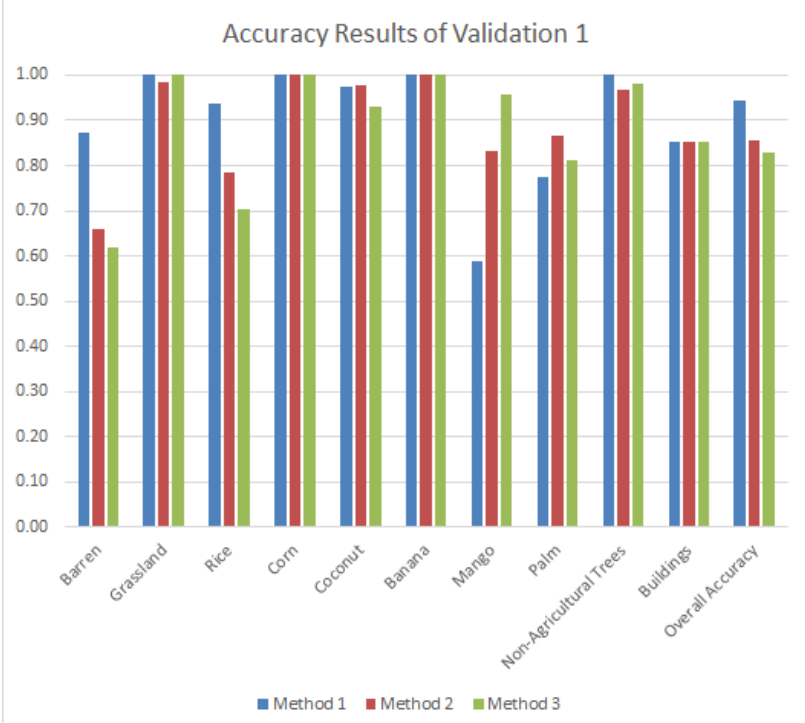

Figure 9. Accuracy Results using Validation Sample Set 1

Table 2. Accuracy Results using Validation Sample Set 2

\begin{tabular}{|l|c|c|c|}
\hline \multirow{4}{*}{ Class } & \multicolumn{3}{c|}{ Method } \\
\cline { 2 - 4 } & $\mathbf{1}$ & $\mathbf{2}$ & $\mathbf{3}$ \\
\hline Barren land & 0.590 & 0.540 & 0.571 \\
\hline Grassland & 1.000 & 1.000 & 1.000 \\
\hline Rice & 0.728 & 0.451 & 0.583 \\
\hline Corn & 1.000 & 1.000 & 1.000 \\
\hline Coconut & 1.000 & 1.000 & 1.000 \\
\hline Banana & 1.000 & 1.000 & 1.000 \\
\hline Mango & 0.651 & 0.941 & 0.891 \\
\hline Palm & 0.877 & 0.951 & 0.865 \\
\hline Non-Agricultural Trees & 1.000 & 0.953 & 0.968 \\
\hline Buildings & 1.000 & 1.000 & 1.000 \\
\hline Overall Accuracy & 0.810 & 0.771 & 0.791 \\
\hline
\end{tabular}




\section{REFERENCES}

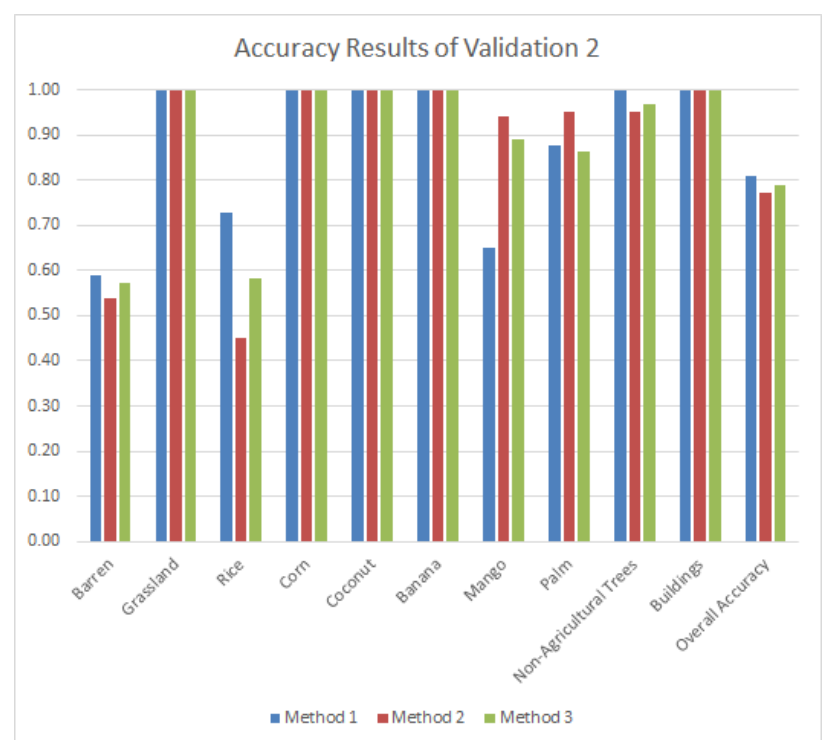

Figure 10. Accuracy Results using Validation Samples Set 2

\section{CONCLUSION AND RECOMMENDATIONS}

Majority of the classes with improved accuracies after applying point cloud-based derivatives belong to Non-Ground classes, specifically, coconut, mango and palm trees. The use of the derivatives on Ground classes in this dataset did not yield satisfactory results. Since there are more classes with decreased accuracy values upon utilizing point cloud-based metrics, the overall accuracy had also shown a decreasing trend in general. Although Method 2 and 3 did not perform well on all classes, we can still utilize them for Non-Ground classes for higher classification accuracy.

For further research, other study sites with varying classes and distribution are highly advisable in order to test the methodology and see if other factors affect the resulting accuracies. Also, try using tree-dominated areas and classify them accordingly. Moreover, other metrics can be computed to determine which point cloud-based derivatives are much more useful in improving the classification results.

\section{ACKNOWLEDGEMENTS}

This study is made possible by the Phil-LiDAR 2 Project funded by the Department of Science and Technology (DOST) and monitored by the Philippine Council for Industry, Energy and Emerging Technology Research and Development (PCIEERD). The Project is also supported by the Department of Agriculture through the Information Technology Center for Agriculture and Fisheries (ITCAF).

The methodologies were developed using the aerial and point cloud data from DREAM/Phil-LiDAR 1 Program.
Alpaydin, E. (2014). Introduction to Machine Learning (Third Edition). Massachusetts: The Massachusetts Institute of Technology Press.

Application, U. o. (2005). Peterson, Birgit; Dubayah, Ralph; Hyde, Peter; Hofton, Michelle; Blair, J. Bryan; Fites-Kaufman, JoAnn. Proceedings of the Seventh Annual Forest Inventory and Analysis Symposium (pp. 193-200). Portland: United States Department of Agriculture, Forest Service, 2007.

Bao, Y., Li, G., Cao, C., Li, X., Zhang, H., He, Q., et al. (2008). Classification of lidar point cloud and generation of $\mathrm{dtm}$ from lidar height and intensity data in forested area. International Archives of the Photogrammetry, Remote Sensing and Spatial Information Sciences, 37 (Part 3B), 313-318.

Burges, C. J. (1998). A Tutorial on Support Vector Machines for Pattern Recognition. Data Mining and Knowledge Discovery 2, 121-167.

Carranza, C., Rollan, T., Tañada, E., Guerrero, J., Jerez, M., \& Blanco, A. (2014). Phil-LIDAR 2 OBIA Training Session Exercises Manual. Nationwide Detailed Resource Assessment using LIDAR (Phil-LIDAR 2) Program Project 1 Agricultural Resources Extraction from LIDAR Surveys (PARMAP). Quezon City, Metro Manila, Philippines.

Cristianini, N., \& Shawe-Taylor, J. (2000). Introduction to Support Vector Machines and Other Kernel-Based Learning Methods. Cambridge: The Press Syndicate of the University of Cambridge.

Disaster Risk and Exposure Assessment for Mitigation (DREAM) Program. (2016). The UP DREAM Program. Retrieved June 29, 2016, from Disaster Risk and Exposure Assessment for Mitigation (DREAM) Program: https://dream.upd.edu.ph/about/

Dy, R. T. (2015, April 20). Why we have to import rice. Retrieved June 23, 2016, from Business World Online: http://www.bworldonline.com/content.php?section=Opinion\&ti tle=why-we-have-to-import-rice $\&$ id $=106455$

Google. (2012, October 16). Cabadbaran, Agusan del Norte, Philippines $9^{\circ} 06^{\prime} 03^{\prime \prime} \mathrm{N}, 1^{\circ} 5^{\circ} 32^{\prime} 26^{\prime \prime} \mathrm{E}$, Eye Alt $23683 \mathrm{ft}$. Retrieved June 22, 2016, from Google Earth Pro v7.1.2.2041: http://www.google.com/earth/index.html

Harrap, R., \& Lato, M. (2006). An Overview of LIDAR for Urban Applications. Kingston, Ontario, Canada.

Hofmann, M. (2006, June 26). Support Vector Machines Kernels and the Kernel Trick. Bamberg, Germany.

Jerez, M. V., Carranza, C. U., Gatdula, N. B., Rollan, T. M., Tañada, E. M., Pagkalinawan, H. M., et al. (2015, December 31). Phil-LiDAR 2 Project 1: Agricultural Resources Extraction from LiDAR Surveys (PARMap) Technical Narrative Report. Quezon City, Metro Manila, Philippines.

Kandrot, S. M. (2013). Coastal Monitoring: A New Approach. Chimera 26: Geographical Journal, University College Cork, 75-89. 
Kecman, V. (2005). Support Vector Machines - An Introduction. In L. Wang, Support Vector Machines: Theory and Applications (pp. 1-47). Heidelberg: Springer-Verlag Berlin Heidelberg.

LiDAR-UK. (n.d.). LiDAR-UK. Retrieved June 2, 2015, from The uses of LiDAR: What applications are there for LiDAR systems?: http://www.lidar-uk.com/usage-of-lidar/

Mattera, D., \& Haykin, S. (1999). Support vector machines for dynamic reconstruction of a chaotic system. In B. Schölkopf, C. J. Burges, \& A. J. Smola, Advances in Kernel Methods: Support Vector Learning (pp. 211-241). Massachusetts: The Massachusetts Institute of Technology Press.

McCue, R. (2009, November 29). A Comparison of the Accuracy of Support Vector Machine and Naive Bayes Algorithms in Spam Classification. Santa Cruz, California, United States of America. Retrieved June 30, 2015, from University of California Santa Cruz: https://classes.soe.ucsc.edu/cmps242/Fall09/proj/RitaMcCueRe port.pdf

National Oceanic and Atmospheric Administration. (2015, May 29). National Oceanic and Atmospheric Administration. Retrieved June 1, 2016, from What is LiDAR?: http://oceanservice.noaa.gov/facts/lidar.html

Philippine Statistics Authority. (2016, May 19). Highlights of the Philippine Population 2015 Census of Population. Retrieved June 23, 2016, from Philippine Statistics Authority: https://www.psa.gov.ph/content/highlights-philippinepopulation-2015-census-population

Provincial Agricultural Office of Agusan del Norte. (n.d.). Agricultural Crops. Retrieved June 20, 2016, from Official Website of Agusan del Norte: http://www.agusandelnorte.gov.ph/index.php/socio-ecoprofile/economic-profile/agricultural-crops

Qiao, J.-G., \& Zhang, Y.-H. (2009). Texture-Based LiDAR Gray Image Segmentation using Artificial Neural Network. Guangzhou, China.

Roli, F., \& Fumera, G. (2001). Support vector machines for remote-sensing. Proceedings of the International Society for Optics and Photonics, Vol. 4170, 160-166.

Schultz, T., \& Kindlmann, G. L. (2013). Open-Box Spectral Clustering: Applications to Medical Image Analysis. IEEE Computer Society.

scikit-learn.org. (n.d.). RBF SVM parameters. Retrieved 2014, from scikit-learn.org: http://scikitlearn.org/stable/auto_examples/svm/plot_rbf_parameters.html

Waske, B., \& Benediktsson, J. A. (2007). Fusion of Support Vector Machines for Classification of Multisensor Data. IEEE Transactions on GeoScience and Remote Sensing, Volume 45, 3858-3866.

Zhang, J., Lin, X., \& Ning, X. (2013). SVM-Based Classification of Segmented Airborne LiDAR Point Clouds in Urban Areas. Remote Sensing (ISSN 2072-4292): Advances in Mobile Laser Scanning and Mobile Mapping, 3749-3775. 